\title{
GEOMETRIC ALGEBRA, DIRAC WAVEFUNCTIONS AND BLACK HOLES
}

\author{
A.N. LASENBY AND C.J.L. DORAN \\ Astrophysics Group \\ Cavendish Laboratory \\ Madingley Road \\ Cambridge CBз OHE, U.K.
}

\begin{abstract}
In this contribution we describe some applications of geometric algebra to the field of black hole physics. Our main focus is on the properties of Dirac wavefunctions around black holes. We show the existence of normalised bound state solutions, with an associated decay rate controlled by an imaginary contribution to the energy eigenvalue. This is attributable to the lack of Hermiticity caused by a black hole singularity. We also give a treatment of the Feynman scattering problem for fermions interacting with black holes that we believe is new, and produces an analogue of the Mott scattering formula for the gravitational case. Throughout, the consistent application of geometric algebra simplifies the mathematical treatment and aids understanding by focusing attention on observable quantities. We finish with a brief review of recent work on the effects of torsion in quadratic theories of gravity. This work demonstrates that a free torsion field can play a significant role in cosmology.
\end{abstract}

\section{Introduction}

This contribution provides a broad survey of a number of recent advances in the application of geometric algebra to quantum physics and gravitation. The bulk of the presentation deals with the quantum properties of fermions in a black hole background. We address two related problems. The first question is whether a spectrum of bound state solutions exists around a black hole. The non-relativistic limit of the Dirac equation suggests that this should be the case, and indeed this is borne out by the full general relativistic theory. The relativistic treatment contains a novel feature, however, which complicates the search for bound states. The Hamilto- 
nian representing a Dirac fermion in a Schwarzschild black hole background is not Hermitian as the singularity acts as a density sink. This means that the eigenspectrum consists of isolated complex energies. The equations are too complicated to admit an analysis in terms of known special functions, so these eigenvalues can only be found by a numerical search. Fortunately, for a range of masses, the real parts of the energies lie close to their nonrelativistic values, which simplifies the search process. The imaginary contribution to the energy is responsible for orbital decay and implies that the bound states have finite lifetimes. This provides a novel, physical picture of what happens as a particle falls into a black hole. Some of the questions raised by this work are discussed here.

The second main application is to the scattering problem for black holes. This has been discussed by many authors, both in a classical and quantum context [1]-[6]. Here we tackle the problem in terms of straightforward Feynman propagator theory. Remarkably, this approach does not appear to have been tried before. The approach is suggested naturally by the geometric algebra form of the Dirac equation in a Schwarzschild background, when cast in Newtonian gauge form. The name reflects the fact that many equations take on a Newtonian form if expressed in this gauge, though of course all physical predictions are gauge invariant. The result of this approach is that the effects of the black hole can be described by a single vertex factor in momentum space. This factor has the unusual property of vanishing on-shell, so the gravitational cross-section involves a secondorder process (in the Newtonian gauge). The calculation can be reproduced in other gauges, and in all cases the cross-section, to leading order in the black hole mass, is

$$
\frac{d \sigma}{d \Omega_{f}}=\frac{(G M)^{2}}{4 \beta^{4} \sin ^{4}(\theta / 2)}\left(1+2 \beta^{2}-3 \beta^{2} \sin ^{2} \frac{\theta}{2}+\beta^{4}-\beta^{4} \sin ^{2} \frac{\theta}{2}\right),
$$

where $\beta=|\boldsymbol{p}| / E$. The cross-section does not depend on the particle mass, which embodies the equivalence principle at the quantum level. The lowvelocity limit recovers the standard Rutherford formula. The full result is the gravitational analogue of the Mott formula for the Coulomb scattering cross-section.

\section{Geometric Algebra}

Throughout this paper we consistently apply the mathematical language of geometric algebra. Though this does not affect any of the physical predictions, it does considerably assist in the understanding and solution of the mathematics encountered. The foundations of geometric algebra (GA) were laid in the 19th Century by Hamilton and Grassmann (Fig. 1). Clif- 


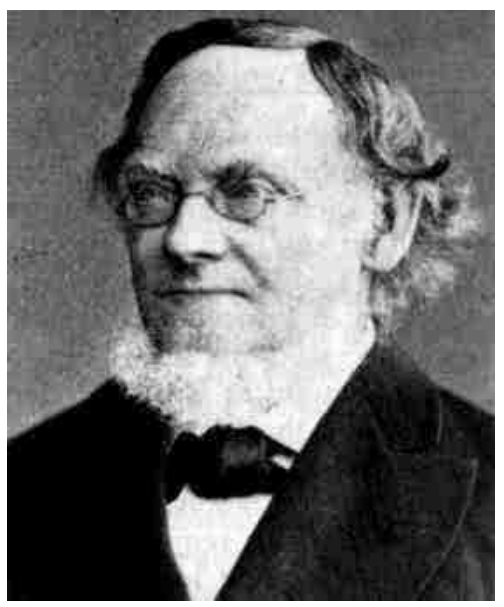

Figure 1. Hermann Gunther Grassmann (1809-1877), born in Stettin, Germany (now Szczecin, Poland). He worked as a schoolteacher in Germany, and later established a significant reputation in linguistics. The mathematical system he introduced, Grassmann algebra, is now an integral part of differential geometry and theoretical physics.

ford (Fig. 2) then unified their work by showing how Hamilton's quaternion algebra could be included in Grassmann's scheme through the introduction of a new, geometric product. The resulting algebra is known as a Clifford algebra, though when used in its intended, geometric role most practitioners prefer Clifford's original name of geometric algebra. The algebra was not immediately adopted by physicists, as it became tainted by its association with quaternions. These ran into problems due to their muddled handling of reflections and rotations, though ironically it is the embedding of quaternions within GA which solves all of these difficulties! Clifford algebra was rediscovered by Pauli and Dirac for use in the quantum theory of spin, and then developed further by mathematicians in the $50 \mathrm{~s}$ and $60 \mathrm{~s}$. It was reintroduced in the guise of geometric algebra to physics in the 70s by David Hestenes, who is largely responsible for the modern form of the subject.

At its most fundamental level, geometric algebra is a mathematical language for directly encoding geometric primitives - points, lines, planes, volumes, circles, spheres etc. Geometric algebra provides a new product for vectors which generalizes complex analysis to arbitrary dimensions. The key to the development of the algebra was Grassmann's introduction of the outer product in the 'Lineale Ausdehnungslehre' of 1844. This product encodes an oriented plane segment via an antisymmetric 'wedge product' $a \wedge b$. Antisymmetry in encoded mathematically in the statement

$$
a \wedge b=-b \wedge a
$$



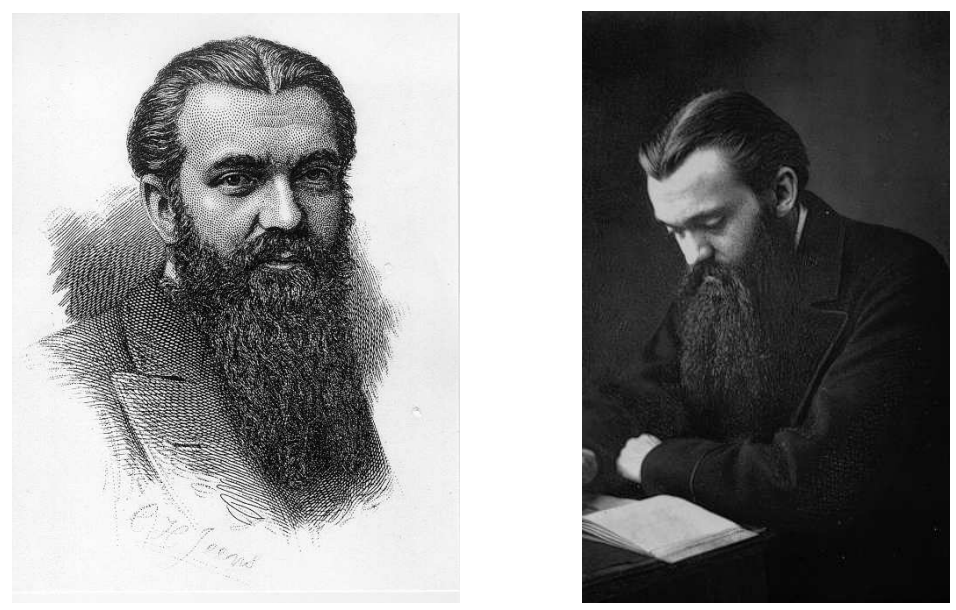

Figure 2. William Kingdon Clifford 1845-1879. Mathematician and philosopher. He died tragically young at the age of only 33 .

which implies that $a \wedge a=0$. For example, suppose that $e_{1}, e_{2}$ are a pair of orthonormal basis vectors in two dimensions. With vectors $a=a_{1} e_{1}+a_{2} e_{2}$ and $b=b_{1} e_{1}+b_{2} e_{2}$, we find that

$$
\begin{aligned}
a \wedge b & =a_{1} b_{2} e_{1} \wedge e_{2}+a_{2} b_{1} e_{2} \wedge e_{1} \\
& =\left(a_{1} b_{2}-b_{2} a_{1}\right) e_{1} \wedge e_{2} .
\end{aligned}
$$

The coefficient here is seen to represent the area of the parallelogram defined by the vectors, and the bivector term $e_{1} \wedge e_{2}$ defines an orientation. Grassmann extended the outer product to arbitrary dimensions and grades. The latter term refers to the number of vectors in each outer product. The total antisymmetry ensures that the outer product of a set of linearly-dependent vectors must vanish. Each step up in grade is therefore a step up in the spatial dimension of the object of interest.

Clifford's great insight was to realise that Grassmann's outer product can be combined with the (symmetric) inner product to define a single product

$$
a b=a \cdot b+a \wedge b .
$$

Clifford called this the geometric product. It satisfies the usual axioms of associativity and distributivity (over addition). Clearly it is not commutative, and the separate scalar (inner) and outer products can be obtained from

$$
a \cdot b=\frac{1}{2}(a b+b a), \quad a \wedge b=\frac{1}{2}(a b-b a) .
$$


These axioms are sufficient to build up an extremely rich algebra. For example, in two dimensions the algebra is spanned by the set

$$
\begin{array}{ccc}
1 & \left\{e_{1}, e_{2}\right\} & e_{1} \wedge e_{2} \\
1 \text { scalar } & 2 \text { vectors } & 1 \text { bivector. }
\end{array}
$$

As the basis vectors are orthogonal, the bivector $e_{1} \wedge e_{2}$ can be written in any of the equivalent forms:

$$
e_{1} \wedge e_{2}=e_{1} e_{2}=-e_{2} \wedge e_{1}=-e_{2} e_{1} .
$$

It follows that the square of the bivector satisfies

$$
\left(e_{1} \wedge e_{2}\right)^{2}=\left(e_{1} e_{2}\right)\left(-e_{2} e_{1}\right)=-e_{1} e_{2} e_{2} e_{1}=-1,
$$

so the unit bivector squares to minus 1! Furthermore, it is responsible for generating $\pi / 2$ rotations in the plane. One can clearly see that the geometric algebra of the Euclidean plane naturally incorporates complex analysis, with the geometric product taking on the role of the complex product.

Similarly, in three dimensions the full algebra is spanned by the set

$$
\begin{array}{cccc}
1 & \left\{\boldsymbol{\sigma}_{i}\right\} & \left\{\boldsymbol{\sigma}_{i} \wedge \boldsymbol{\sigma}_{j}\right\} & I=\boldsymbol{\sigma}_{1} \boldsymbol{\sigma}_{2} \boldsymbol{\sigma}_{3} \\
\text { 1 scalar } & 3 \text { vectors } & 3 \text { bivectors } & 1 \text { trivector. }
\end{array}
$$

(The reason for the notation will emerge shortly.) Each grade encodes a geometrically significant object in three dimensions. The highest grade object, $I$, is called the pseudoscalar. In three dimensions it has negative square, $I^{2}=-1$. The pseudoscalar maps vectors to bivectors, and vice-versa. For the bivectors we can write

$$
\sigma_{1} \sigma_{2}=I \sigma_{3}, \quad \sigma_{2} \sigma_{3}=I \sigma_{1}, \quad \sigma_{3} \sigma_{1}=I \sigma_{2} .
$$

It follows that each bivector squares to -1 . If we set

$$
\boldsymbol{i}=I \boldsymbol{\sigma}_{1}, \quad \boldsymbol{j}=-I \boldsymbol{\sigma}_{2}, \quad \boldsymbol{k}=I \boldsymbol{\sigma}_{3}
$$

we find that $\boldsymbol{i}, \boldsymbol{j}, \boldsymbol{k}$ satisfy

$$
\boldsymbol{i}^{2}=\boldsymbol{j}^{2}=\boldsymbol{k}^{2}=\boldsymbol{i} \boldsymbol{j} \boldsymbol{k}=-1 .
$$

This are the defining relations of the quaternion algebra, which is naturally embedded in the algebra of 3D space. The embedding is important, as it separates the roles of vectors and quaternions (bivectors). This is essential for the correct handling of reflections and rotations.

Further information can be found in a number of introductions to geometric algebra. See for example the papers by Hestenes $[7,8,9]$ and 
Vold $[10,11]$ and the books $[12,13,14,15]$. See also [16] for a complete set of lecture notes and further resources.

\section{Spacetime Algebra}

The applications in this paper are mainly developed in the geometric algebra of spacetime. This is called the spacetime algebra or STA. We let the set $\left\{\gamma_{\mu}\right\}$ denote an orthogonal frame of vectors for spacetime, where $\gamma_{0}^{2}=1$, and $\gamma_{k}^{2}=-1$ for $k=1,2,3$. The inner product for these is summarised by

$$
\gamma_{\mu} \cdot \gamma_{\nu}=\frac{1}{2}\left(\gamma_{\mu} \gamma_{\nu}+\gamma_{\nu} \gamma_{\mu}\right)=\eta_{\mu \nu}=\operatorname{diag}(+---) .
$$

These are the defining relations of the Dirac matrix algebra, so the Dirac matrices form a representation for the STA. We do not use the matrix representation directly in calculations, though, as it is invariably slower. For example, one can end up calculating the square of a $4 \times 4$ complex matrix, when all this encodes is the result $\gamma_{0}^{2}=1$. Also, the matrices are naturally defined over the complex field, whereas we are interested in the real STA.

Repeated multiplication of the basis vector builds up a complete basis for the STA. This has $2^{4}=16$ elements, and is written

$$
\begin{array}{ccccc}
1 & \left\{\gamma_{\mu}\right\} & \left\{\boldsymbol{\sigma}_{k}, I \boldsymbol{\sigma}_{k}\right\} & \left\{I \gamma_{\mu}\right\} & I \\
\text { scalar } & \text { vectors } & \text { bivectors } & \text { pseudovectors } & \text { pseudoscalar. }
\end{array}
$$

The bivectors $\boldsymbol{\sigma}_{k}$ are defined by

$$
\boldsymbol{\sigma}_{k}=\gamma_{k} \gamma_{0}
$$

Their algebra is isomorphic to that of Euclidean 3-space, since

$$
\boldsymbol{\sigma}_{j} \cdot \boldsymbol{\sigma}_{k}=\frac{1}{2}\left(\boldsymbol{\sigma}_{j} \boldsymbol{\sigma}_{k}+\boldsymbol{\sigma}_{k} \boldsymbol{\sigma}_{j}\right)=\delta_{j k}
$$

These define a set of basis vectors for the rest frame defined by $\gamma_{0}$. Different timelike vectors define different relative vectors, and hence a different spacetime split of the bivectors in the STA. The spacetime pseudoscalar is again denoted $I$, and is given by

$$
I=\boldsymbol{\sigma}_{1} \boldsymbol{\sigma}_{2} \boldsymbol{\sigma}_{3}=\gamma_{1} \gamma_{0} \gamma_{2} \gamma_{0} \gamma_{3} \gamma_{0}=\gamma_{0} \gamma_{1} \gamma_{2} \gamma_{3}
$$

It follows that $I^{2}=-1$ and, in spacetime, $I$ anticommutes with all vectors.

For a general vector $a$ we write the spacetime split with $\gamma_{0}$ as

$$
a \gamma_{0}=a_{0}+\boldsymbol{a}
$$


where

$$
a_{0}=a \cdot \gamma_{0}, \quad \boldsymbol{a}=a \wedge \gamma_{0} .
$$

Relative vectors are denoted in bold, to distinguish them from spacetime vectors. Of course, in the full STA, relative vectors are treated as bivectors. For example, the momentum $p$ is split according to

$$
p \gamma_{0}=p \cdot \gamma_{0}+p \wedge \gamma_{0}=E+\boldsymbol{p} .
$$

where $E$ is the energy measured in the $\gamma_{0}$ frame. We immediately recover the Lorentz invariant scalar from

$$
p^{2}=p \gamma_{0} \gamma_{0} p=(E+\boldsymbol{p})(E-\boldsymbol{p})=E^{2}-\boldsymbol{p}^{2} .
$$

(We use natural units throughout, so $c=\epsilon_{0}=\hbar=1$.) Velocity 4-vectors are invariably normalised so that $v^{2}=1$. For these the relative velocity in the $\gamma_{0}$ frame is defined by

$$
\boldsymbol{v}=\frac{v \wedge \gamma_{0}}{v \cdot \gamma_{0}}
$$

so that we have

$$
v \gamma_{0}=\left(v \cdot \gamma_{0}\right)(1+\boldsymbol{v})=\gamma(1+\boldsymbol{v})
$$

where $\gamma=\left(1-\boldsymbol{v}^{2}\right)^{-1 / 2}$ is the Lorentz factor.

A general multivector in the STA can contain a mixture of grades. It is useful to have a compact notation to denote the result of projecting out terms of a given grade. We write this as $\langle A\rangle_{r}$, which returns the grade- $r$ terms in $A$. For the scalar part $(r=0)$ we drop the subscript and just write

$$
\langle A\rangle=\langle A\rangle_{0} .
$$

Multivectors containing terms of only a single grade are called homogeneous. For these the inner and outer products extend straightforwardly. For multivectors $A_{r}$ and $B_{s}$ of grade $r$ and $s$ respectively, we can write

$$
\begin{aligned}
A_{r} \cdot B_{s} & =\left\langle A_{r} B_{s}\right\rangle_{|r-s|} \\
A_{r} \wedge B_{s} & =\left\langle A_{r} B_{s}\right\rangle_{r+s} .
\end{aligned}
$$

In the case where $r=1$, so that $a=A_{r}$ is a vector, we have the relation

$$
a B_{s}=a \cdot B_{s}+a \wedge B_{s} .
$$

The symmetry of both the inner and outer product alternate with increasing grade of $B_{s}$,

$$
\begin{aligned}
a \cdot B_{s} & =\left\langle a B_{s}\right\rangle_{s-1}=\frac{1}{2}\left(a B_{s}-(-1)^{s} B_{s} a\right) \\
a \wedge B_{s} & =\left\langle a B_{s}\right\rangle_{s+1}=\frac{1}{2}\left(a B_{s}+(-1)^{s} B_{s} a\right) .
\end{aligned}
$$


We adopt the convention that, in the absence of brackets, inner and outer products are performed before geometric products.

An important operation in GA is that of reversion. We write the reverse of $A$ as $\widetilde{A}$, which reverses all the vector products making up the multivector. This has the property that

$$
(A B)^{\sim}=\widetilde{B} \widetilde{A} .
$$

Given a general multivector

$$
M=\alpha+a+B+I b+I \beta,
$$

where $\alpha$ and $\beta$ are scalars, $a$ and $b$ are vectors and $B$ is a bivector, the reverse satisfies

$$
\widetilde{M}=\alpha+a-B-I b+I \beta .
$$

Lorentz transformations are spacetime rotations and can be performed by the use of a rotor. A rotor $R$ is an even-grade element of the STA satisfying

$$
R \widetilde{R}=\widetilde{R} R=1 .
$$

A proper orthochronous Lorentz transformation of a vector $a$ can be written

$$
a \mapsto a^{\prime}=R a \widetilde{R} .
$$

It can be shown that all proper orthochronous Lorentz transformations can be written in this way. Since $R$ and $-R$ encode the same transformation, rotors form a double cover of the (restricted) Lorentz group. Any rotor can be written in the form

$$
R= \pm \exp (-B / 2)
$$

where $B$ is a bivector. The bivectors form the Lie algebra of the Lorentz group, and directly encode the spacetime plane(s) in which the transformation is performed. The same double-sided transformation law applies to multivectors representing geometric objects. For example, the plane $a \wedge b$ transforms to

$$
a \wedge b \mapsto(R a \widetilde{R}) \wedge(R b \widetilde{R})=\frac{1}{2}(R a \widetilde{R} R b \widetilde{R}-R b \widetilde{R} R a \widetilde{R})=R a \wedge b \widetilde{R} .
$$

The fact that general multivectors transform in this simple manner is a considerable improvement over matrix-based techniques for carrying out Lorentz transformations.

The main ingredient in field theory in the STA is the vector derivative $\nabla$. If $x^{\mu}$ denote the coordinates in each of the $\gamma_{\mu}$ directions, we write

$$
\nabla=\gamma^{\mu} \frac{\partial}{\partial x^{\mu}}=\gamma_{0} \frac{\partial}{\partial t}+\gamma^{i} \frac{\partial}{\partial x^{i}} .
$$


The spacetime split of $\nabla$ goes as

$$
\nabla \gamma_{0}=\partial_{t}-\nabla
$$

where $\boldsymbol{\nabla}=\boldsymbol{\sigma}_{i} \partial_{i}$ is the vector derivative in the relative space picked out by $\gamma_{0}$. The spacetime vector derivative $\nabla$ provides a remarkably compact encoding of the Maxwell equations. We combine the electric and magnetic fields into spacetime bivector $F=\boldsymbol{E}+I \boldsymbol{B}$. The Maxwell equations can then be written as

$$
\nabla F=J
$$

where $J$ is the current. This is not merely a cosmetic exercise. The vector derivative $\nabla$ is directly invertible, which provides a number of new techniques for solving the Maxwell equations.

\section{Quantum Theory}

The fact that the Pauli and Dirac matrix algebras are representations of the geometric algebras of real space and spacetime suggests that GA is a natural tool for studying quantum theory. This is indeed the case. Both Pauli and Dirac spinors can be handled in the real geometric algebras of space and spacetime, and this provides a number of insights into their geometric roles. We start with the case of non-relativistic spinors. A Pauli column spinor $\psi^{a}$ can be placed in a direct $1 \leftrightarrow 1$ correspondence with an element of the even subalgebra of 3D geometric algebra as follows:

$$
\psi^{a}=\left(\begin{array}{r}
a^{0}+i a^{3} \\
-a^{2}+i a^{1}
\end{array}\right) \quad \leftrightarrow \quad \psi=a^{0}+a^{k} I \boldsymbol{\sigma}_{k} .
$$

The actions of the quantum operators $\left\{\hat{\sigma}_{k}\right\}$, and the unit imaginary $i$, are replaced by the operations

$$
\begin{aligned}
\hat{\sigma}_{k}|\psi\rangle & \leftrightarrow \boldsymbol{\sigma}_{k} \psi \boldsymbol{\sigma}_{3} \quad(k=1 \ldots 3) \\
i|\psi\rangle & \leftrightarrow \psi \boldsymbol{I} \boldsymbol{\sigma}_{3} .
\end{aligned}
$$

A feature of this scheme is that the complex structure is taken over entirely by the bivector $I \boldsymbol{\sigma}_{3}$, acting to the right of $\psi$.

Every calculation that can be performed with the column spinor $\psi^{a}$ can also be performed with the even element $\psi$, and in practice the latter approach is usually easier. One reason for this is the natural decomposition of $\psi$ into a density term and a rotor:

$$
\psi=\rho^{1 / 2} R,
$$

where

$$
\rho=\psi \widetilde{\psi} .
$$


For example, the spin vector $s$ has components

$$
s_{k}=\left\langle\psi\left|\hat{\sigma}_{k}\right| \psi\right\rangle \text {. }
$$

In terms of GA these become

$$
s_{k}=\left\langle\widetilde{\psi} \boldsymbol{\sigma}_{k} \psi \boldsymbol{\sigma}_{3}\right\rangle=\boldsymbol{\sigma}_{k} \cdot\left(\psi \boldsymbol{\sigma}_{3} \widetilde{\psi}\right) .
$$

It follows that all of the components of the spin vector can be summarised in the single expression

$$
\boldsymbol{s}=\psi \boldsymbol{\sigma}_{3} \widetilde{\psi}=\rho R \boldsymbol{\sigma}_{3} \widetilde{R} .
$$

The $3 \mathrm{D}$ rotor $R$ is therefore an instruction to rotate the fixed $\boldsymbol{\sigma}_{3}$ vector onto the observable spin vector. This establishes a natural link with the description of a rotating rigid body [15, 17].

The normalised observables define the unit spin vector

$$
\hat{\boldsymbol{s}}=\frac{\psi \boldsymbol{\sigma}_{3} \widetilde{\psi}}{\rho}=R \boldsymbol{\sigma}_{3} \widetilde{R} .
$$

This defines a unit vector which is usually represented as a point on the Bloch sphere. The quantum density matrix is also easily encoded. For normalised pure states the density matrix is defined by

$$
\hat{\rho}=|\psi\rangle\langle\psi| .
$$

This maps directly to the equivalent multivector

$$
\hat{\rho}=\psi \frac{1}{2}\left(1+\boldsymbol{\sigma}_{3}\right) \widetilde{\psi}=\frac{1}{2}(1+\hat{\boldsymbol{s}}) .
$$

For mixed states we simply sum the density matrices and normalise, so that

$$
\hat{\rho}=\frac{1}{2}(1+\boldsymbol{P}), \quad \boldsymbol{P}^{2} \leq 1 .
$$

This is sufficient to encode single particle quantum theory, but what we really need is a version of multiparticle quantum mechanics. Surprisingly, this requires the full, relativistic theory.

A similar construction for Pauli spinors can be applied to Dirac spinors. A Dirac spinor has 8 real components, and these are placed in a $1 \leftrightarrow 1$ correspondence with a even-grade element of the STA as follows:

$$
|\psi\rangle=\left(\begin{array}{c}
|\phi\rangle \\
|\eta\rangle
\end{array}\right) \leftrightarrow \psi=\phi+\eta \boldsymbol{\sigma}_{3} .
$$


Here $|\phi\rangle$ and $|\eta\rangle$ are two-component spinors, and $\phi$ and $\eta$ are their Paulieven equivalents. The action of the operators $\left\{\hat{\gamma}_{\mu}, \hat{\gamma}_{5}, i\right\}$ becomes

$$
\begin{aligned}
& \hat{\gamma}_{\mu}|\psi\rangle \quad \leftrightarrow \quad \gamma_{\mu} \psi \gamma_{0} \quad(\mu=0, \ldots, 3) \\
& i|\psi\rangle \quad \leftrightarrow \quad \psi I \sigma_{3} \\
& \hat{\gamma}_{5}|\psi\rangle \quad \leftrightarrow \quad \psi \boldsymbol{\sigma}_{3} .
\end{aligned}
$$

With these relations one can immediately write down a form of the Dirac equation in the STA as

$$
\nabla \psi I \boldsymbol{\sigma}_{3}-e A \psi=m \psi \gamma_{0}
$$

where $A$ is the electromagnetic vector potential and $m$ is the mass. This equation is entirely equivalent to the standard matrix form of the Dirac equation, even though it is written entirely in the real STA.

The main observables in Dirac theory are also simply encoded now. For example, the current is defined by

$$
J^{\mu}=\left\langle\psi\left|\hat{\gamma}^{\mu}\right| \psi\right\rangle \quad \leftrightarrow \quad\left\langle\widetilde{\psi} \gamma^{\mu} \psi \gamma_{0}\right\rangle=\gamma^{\mu} \cdot\left(\psi \gamma_{0} \widetilde{\psi}\right)
$$

We can therefore reconstitute the vector $J=J^{\mu} \gamma_{\mu}$ to obtain

$$
J=\psi \gamma_{0} \widetilde{\psi}
$$

The remaining observables have equally simple expressions [18]. Further insight into the role of a spinor is provided by first writing

$$
\psi \widetilde{\psi}=\rho e^{I \beta}
$$

so that we can set

$$
\psi=\rho^{1 / 2} e^{I \beta / 2} R .
$$

The object $R$ satisfies $R \widetilde{R}=1$, so is a spacetime rotor. We now find that

$$
J=\rho R \gamma_{0} \widetilde{R}=\rho v,
$$

so the velocity $v$ is obtained from a boost of the $\gamma_{0}$ vector onto the observable. The boost is controlled by the rotor $R$.

Now suppose we wish to extend to describe an $n$-particle quantum state. We construct a $4 n$-dimensional relativistic configuration space, spanned by the vectors $\left\{\gamma_{\mu}^{a}\right\}$, where $\mu=0 \ldots 3$ labels the spacetime vector, and $a=1 \ldots n$ labels the particle space. Vectors from distinct particle spaces are orthogonal, so we have

$$
\gamma_{\mu}^{a} \cdot \gamma_{\nu}^{b}=\eta_{\mu \nu} \delta^{a b}
$$


With this algebra one can construct a multiparticle Dirac equation, which provides a simple, geometric encoding of Pauli antisymmetrisation [18]. If we now apply a spacetime split in one space we obtain the relative vectors

$$
\boldsymbol{\sigma}_{j}^{a}=\gamma_{j}^{a} \gamma_{0}^{a}, \quad j=1 \ldots 3, \quad a=1 \ldots n .
$$

Bivectors from distinct spaces commute, as can be seen from

$$
\begin{aligned}
\boldsymbol{\sigma}_{i}^{a} \boldsymbol{\sigma}_{j}^{b} & =\gamma_{i}^{a} \gamma_{0}^{a} \gamma_{j}^{b} \gamma_{0}^{b} \\
& =\gamma_{i}^{a} \gamma_{j}^{b} \gamma_{0}^{b} \gamma_{0}^{a} \\
& =\gamma_{j}^{b} \gamma_{0}^{b} \gamma_{i}^{a} \gamma_{0}^{a}=\boldsymbol{\sigma}_{j}^{b} \boldsymbol{\sigma}_{i}^{a} \quad(a \neq b) .
\end{aligned}
$$

It follows that the geometric product of elements of even grade from different spaces is equivalent to the tensor product. This makes it a straightforward exercise to construct multiparticle quantum states, and all that is ever required is the geometric product! This idea is now the basis for a wide range of applications, including quantum information theory and NMR [19, 20, 21].

\section{Gravitation}

The STA is the geometric algebra of (flat) Lorentzian spacetime. This might appear to pose a problem for its continued application in general relativity, where spacetime becomes curved. This turns out not to be the case. The solution is to work with a gauge theory formulation of gravity. The first satisfactory gauge treatment of gravity was formulated by Kibble in 1961 [22]. The gauge theory approach leads naturally to an extended version of general relativity (GR) known as a spin-torsion theory [23] though, in the absence of macroscopic spin, the equations reduce to those of GR. In [24] the gauge theory treatment was reconsidered in the STA framework. It was shown that gravitation can be developed as a gauge theory built on arbitrary finite transformations. And, unlike Kibble's earlier theory, the combination of the gauge treatment and STA produces a theory which is conceptually and computationally simpler than GR. This theory is known as gauge theory gravitation, or GTG.

The gauge theory of gravitation requires the introduction of two gauge fields. The first is a position-dependent linear function $\overline{\mathrm{h}}(a)$, or $\overline{\mathrm{h}}(a, x)$. This is linear in its vector argument $a$, and is a general non-linear function of the position vector $x=x^{\mu} \gamma_{\mu}$. This gauge field ensures that the equations remain covariant under arbitrary, nonlinear displacement. Suppose that the vector field $J(x)$ is defined by

$$
J=\overline{\mathrm{h}}(\nabla \phi) .
$$


Then, if we replace $\phi(x)$ by $\phi^{\prime}(x)=\phi\left(x^{\prime}\right)$, and transform $\overline{\mathrm{h}}$ accordingly, $J(x)$ transforms simply to $J^{\prime}(x)=J\left(x^{\prime}\right)$. By inserting the $\overline{\mathrm{h}}$ field at various points in STA field equations, we can construct equations which remain covariant under arbitrary displacements.

The second gauge field is denoted $\Omega(a)=\Omega(a, x)$, and is a bivectorvalued linear function of its argument $a$. The position-dependence of $\Omega(a)$ is also generally non-linear. The bivector field $\Omega(a)$ is the connection for the gauge group of Lorentz transformations. Recall that a Lorentz transformation can be written as $a \mapsto R a \widetilde{R}$. Suppose now that all fields are subject to a local rotation generated by the position dependent rotor $R$. The gauge fields transform as:

$$
\overline{\mathrm{h}}(a) \mapsto \overline{\mathrm{h}}^{\prime}(a)=R \overline{\mathrm{h}}(a) \widetilde{R},
$$

and

$$
\Omega(a) \mapsto \Omega^{\prime}(a)=R \Omega(a) \widetilde{R}-2 a \cdot \nabla R \widetilde{R} .
$$

The gauge fields ensure that equations can remain covariant under local rotations, as well as arbitrary displacements. For example, the Dirac equation generalises to

$$
\overline{\mathrm{h}}\left(\gamma^{\mu}\right) D_{\mu} \psi I \boldsymbol{\sigma}_{3}=m \psi \gamma_{0},
$$

where

$$
D_{\mu} \psi=\left(\partial_{\mu}+\frac{1}{2} \Omega\left(\gamma_{\mu}\right)\right) \psi
$$

The Dirac wavefunction transforms as $\psi \mapsto R \psi$ under rotations. Observables, such as the current $\psi \gamma_{0} \widetilde{\psi}$, then transform as covariant multivectors.

The gravitational field equations can be expressed in various ways. Suppose we introduce an arbitrary set of coordinates $x^{\mu}$ in the STA, with associated coordinate frame $\left\{e_{\mu}\right\}$ and reciprocal frame $\left\{e^{\mu}\right\}$. From these we define

$$
g^{\mu}=\overline{\mathrm{h}}\left(e^{\mu}\right), \quad g_{\mu}=\mathrm{h}^{-1}\left(\mathrm{e}_{\mu}\right) .
$$

(The overbar denotes the adjoint on the linear function $h(a)$ ). In terms of these the metric is defined by

$$
g_{\mu \nu}=g_{\mu} \cdot g_{\nu}
$$

One can proceed to perform all calculations directly from the metric, but this is by no means the best way to work. Instead it is preferable to work with an expanded set of first-order equations. We define

$$
L_{a}=a \cdot \overline{\mathrm{h}}(\nabla)=a \cdot g^{\mu} \partial_{\mu}, \quad \omega(a)=a \cdot g^{\mu} \Omega_{\mu} .
$$

Assuming no torsion is present, the $\overline{\mathrm{h}}(a)$ and $\omega(a)$ gauge fields are related by the bracket identity

$$
\left[L_{a}, L_{b}\right]=L_{c}
$$


where

$$
c=L_{a} b+\omega(a) \cdot b-L_{b} a-\omega(b) \cdot a .
$$

The gravitational field strength is encoded in the Riemann tensor, $\mathcal{R}(a \wedge b)$, which is defined by

$$
\mathcal{R}(a \wedge b)=L_{a} \omega(b)-L_{b} \omega(a)+\omega(a) \times \omega(b)-\omega(c),
$$

with $c$ determined by equation (70). The Ricci and Einstein tensors and the Ricci scalar are defined by

$$
\begin{array}{rrrl}
\text { Ricci Tensor: } & \mathcal{R}(b)=\gamma_{\mu} \cdot \mathcal{R}\left(\gamma^{\mu} \wedge b\right) \\
\text { Ricci Scalar: } & \mathcal{R}=\gamma_{\mu} \cdot \mathcal{R}\left(\gamma^{\mu}\right) \\
\text { Einstein Tensor: } & \mathcal{G}(a)=\mathcal{R}(a)-\frac{1}{2} a \mathcal{R} .
\end{array}
$$

\section{Spherical Systems}

Our analysis of the effects of a spherical black hole starts with the Schwarzschild solution in the following form:

$$
d s^{2}=d t^{2}-\left(d r+\left(\frac{2 G M}{r}\right)^{1 / 2} d t\right)^{2}-r^{2} d \Omega^{2} .
$$

Here the time coordinate $t$ is the proper time of an observer freely falling from rest at infinity. This simple form of the Schwarzschild solution was first given by Painlevé and Gullstrand (see [25]). The solution is well-behaved at the horizon, and has a number of other attractive properties, but it has been strangely neglected. In terms of the $\bar{h}$ function, the solution is generated by

$$
\overline{\mathrm{h}}(a)=a-\sqrt{2 M / r} a \cdot e_{r} e_{t} .
$$

We call this particular gauge choice the 'Newtonian gauge', due to its simple Newtonian properties (these are discussed further in [24]).

We use this gauge to put the Dirac equation into Hamiltonian form. The derivation is particularly clear in Cartesian coordinates. The metric is now

$$
d s^{2}=\eta_{\mu \nu} d x^{\mu} d x^{\nu}-\frac{G M}{r} d t^{2}-\frac{2}{r}\left(\frac{2 G M}{r}\right)^{1 / 2} a_{\mu} d t d x^{\mu}
$$

where $a_{\mu}=(0, x, y, z)$. The $g_{\mu}$ vectors are now given by

$$
g_{0}=\gamma_{0}+\left(\frac{2 G M}{r}\right)^{1 / 2} \frac{x_{i}}{r} \gamma_{i}, \quad g_{i}=\gamma_{i} \quad(i=1,2,3)
$$


and one can check easily that $g_{\mu} \cdot g_{\nu}=g_{\mu \nu}$. The $g_{\mu}$ vectors generate a Dirac equation of the form

$$
\nabla \psi I \boldsymbol{\sigma}_{3}-\left(\frac{2 G M}{r}\right)^{1 / 2} \gamma_{0}\left(\partial_{r} \psi+3 /(4 r) \psi\right) I \boldsymbol{\sigma}_{3}=m \psi \gamma_{0}
$$

The full relativistic wave equation in a black hole background therefore picks up a single interaction term:

$$
H_{I} \psi=\left(\frac{2 G M}{r}\right)^{1 / 2} \hbar\left(\partial_{r} \psi+3 /(4 r) \psi\right) I \boldsymbol{\sigma}_{3} .
$$

(The $\hbar$ has been written explicitly here to emphasise the relation with the standard radial momentum operator $-i \hbar \partial_{r}$.) This is impressively simple, but there is a subtlety connected with the Hermiticity of $H_{I}$. Writing

$$
H_{I}(\psi)=(2 M / r)^{1 / 2} r^{-3 / 4} \partial_{r}\left(r^{3 / 4} \psi\right) I \sigma_{3},
$$

we find that

$$
\begin{aligned}
\int d^{3} x\left\langle\phi^{\dagger} H_{I}(\psi)\right\rangle_{S} & =\sqrt{2 M} \int d \Omega \int_{0}^{\infty} r^{2} d r r^{-5 / 4}\left\langle\phi^{\dagger} \partial_{r}\left(r^{3 / 4} \psi\right) I \boldsymbol{\sigma}_{3}\right\rangle_{S} \\
& =\int d^{3} x\left\langle\left(H_{I}(\phi)^{\dagger} \psi\right\rangle_{S}+\sqrt{2 M} \int d \Omega\left[r^{3 / 2}\left\langle\phi^{\dagger} \psi I \boldsymbol{\sigma}_{3}\right\rangle_{S}\right]_{0}^{\infty}\right.
\end{aligned}
$$

where \langle\rangle$_{S}$ denotes the projection onto the 'complex' 1 and $I \boldsymbol{\sigma}_{3}$ terms, and $\phi^{\dagger}=\gamma_{0} \widetilde{\phi} \gamma_{0}$. For all normalised states the final term in (82) tends to zero as $r \rightarrow \infty$. But it can be shown that wavefunctions tend to the origin as $r^{-3 / 4}$, so the lower limit is finite and $H_{I}$ is therefore not (quite) a Hermitian operator. This immediately rules out the existence of normalisable stationary states with constant real energy, and gives us an insight into the nature of wavefunction decay and collapse into a black hole, which will be important below.

\subsection{THE SCHRÖDINGER LIMIT}

To begin understanding some of the physical properties of this interaction term, we can check that its properties are sensible in the Schrödinger limit. Defining a reduced radial wavefunction $U(r)$ via

$$
\psi=\frac{U}{r} \Phi(\theta, \phi)
$$


one finds that the standard reduction of the Dirac equation to the Schrödinger equation (here in the presence of the interaction term) yields

$$
\frac{d^{2} U}{d r^{2}}-l(l+1) \frac{U}{r^{2}}-\frac{2 m i}{\hbar}\left(\frac{2 G M}{r}\right)^{1 / 2} r^{1 / 4} \frac{d}{d r}\left(U r^{-1 / 4}\right)=-\frac{2 m E}{\hbar^{2}} U
$$

Now the spherically symmetric gravitational problem in the Schrödinger case is formally similar to that of a charged nucleus. In both cases we have an underlying inverse square force. In fact we can manipulate the above equation all the way to complete agreement with the Hydrogen atom problem by carrying out a phase transformation as follows. Define the gravitational Bohr radius

$$
a_{0}=\frac{\hbar^{2}}{G M m^{2}}
$$

and set

$$
U=W \exp \left(i\left(8 r / a_{0}\right)^{1 / 2}\right) .
$$

Then the Schrödinger equation becomes

$$
W^{\prime \prime}-\left(\frac{l(l+1)}{r}-\frac{2}{a_{0} r}-\frac{2 m E}{\hbar^{2}}\right) W=0
$$

which is now identical to the Hydrogen atom case. We can therefore read off the energy level spectrum:

$$
E=-\frac{R_{G}}{n^{2}}
$$

where the gravitational Rydberg is given by

$$
R_{G}=\frac{m}{2}\left(\frac{G M m}{\hbar}\right)^{2}
$$

As we shall see later, these energy levels and associated wavefunctions do provide good approximations for the relativistic solutions in cases where the bulk of the probability density lies well outside the black hole horizon. However, we can see that near the black hole horizon, the Schrödinger approach becomes inconsistent. If we consider the Schrödinger current, we find that it is given by

$$
\frac{\rho \hbar}{m} \nabla\left(\frac{8 r}{a_{0}}\right)^{1 / 2}=\rho\left(\frac{2 G M}{r}\right)^{1 / 2} \hat{\boldsymbol{r}}
$$

where $\hat{\boldsymbol{r}}$ is a unit vector in the radial direction. This implies a velocity associated with the current of $(2 G M / r)^{1 / 2}$ - the same as that of an observer 
freely falling from rest at infinity. The problem, of course, is that this becomes equal to $c$ at the horizon, meaning that the Schrödinger approach becomes inconsistent there. Instead, therefore, we must work with the full Dirac theory.

\subsection{RELATIVISTIC BOUND STATES}

We start by assuming

$$
\psi(x)=\phi(\boldsymbol{x}) \alpha(t)
$$

in equation (79). As usual, the solution of the $t$-equation is

$$
\alpha(t)=\exp \left(-I \sigma_{3} E t\right)
$$

where $E$ is the separation constant. The non-Hermiticity of $H_{I}$ means that $E$ cannot be purely real if $\psi$ is normalisable. The imaginary part of $E$ is determined by equation (82) and, for suitably normalised states, we find that

$$
\operatorname{Im}(E)=-\lim _{r \rightarrow 0} 2 \pi \sqrt{2 M}\left\langle\phi^{\dagger} \phi\right\rangle r^{3 / 2} .
$$

This equation shows that the imaginary part of $E$ is necessarily negative, so the wavefunction decays with time. This is consistent with the fact that the streamlines generated by the conserved current $\psi \gamma_{0} \widetilde{\psi}$ are timelike curves and, once inside the horizon, must ultimately terminate on the origin. However, we can still ask the question of whether a discrete set of complex energy eigenvalues exist. This would then give us a set of spectral levels similar to that in a Hydrogen atom, except with the novelty that each state decays with time, making it a type of resonance.

We now show this does in fact happen, but a crucial question before continuing is whether the energy eigenvalues so obtained are physically meaningful. In the GTG approach, this will be the case if we can show that the values obtained are gauge invariant. In [24], we show that the radial coordinate $r$ is physically well-defined (e.g. it can be experimentally determined by tidal forces) and hence that the full set of gauge transformations compatible with our setup in the spherically symmetric case are:

$$
\begin{aligned}
& t \mapsto t+f(r), \quad \text { position gauge change } \\
& \psi \mapsto R \psi, \quad \text { rotation gauge change }
\end{aligned}
$$

with the rotor $R$ being a function of $\boldsymbol{x}$ only $(R=R(\boldsymbol{x}))$. Suppose that we have a solution in some gauge

$$
\psi(x)=\phi(\boldsymbol{x}) \exp \left(-I \boldsymbol{\sigma}_{3} E t\right),
$$


where $E=E_{r}+I \sigma_{3} E_{i}$. We now carry out a gauge transformation of the above form, obtaining

$$
\begin{aligned}
R \psi\left(x^{\prime}\right) & =R \phi(\boldsymbol{x}) \exp \left(-I \boldsymbol{\sigma}_{3} E(t+f(r))\right) \\
& =R(\boldsymbol{x}) \phi(\boldsymbol{x}) \exp \left(-I \boldsymbol{\sigma}_{3} E t\right) \exp \left(-I \boldsymbol{\sigma}_{3} E f(r)\right)
\end{aligned}
$$

Thus

$$
\partial_{t} R \psi\left(x^{\prime}\right) I \sigma_{3}=R \psi\left(x^{\prime}\right)\left(E_{r}+I \sigma_{3} E_{i}\right)
$$

i.e. the new wavefunction is still an eigenfunction of the evolution operator, with the same eigenvalue. This means $E_{r}$ and $E_{i}$ are gauge invariant, and hence potentially physically observable.

Proceeding with a solution in the Newtonian gauge, we carry out an angular separation via writing

$$
\psi(\boldsymbol{x}, \kappa)= \begin{cases}\psi_{l}^{m} u(r)+\hat{\boldsymbol{r}} \psi_{l}^{m} v(r) I \boldsymbol{\sigma}_{3} & \kappa=l+1 \\ \hat{\boldsymbol{r}} \psi_{l}^{m} u(r) \boldsymbol{\sigma}_{3}+\psi_{l}^{m} i v(r) & \kappa=-(l+1),\end{cases}
$$

where $\kappa$ is a non-zero integer and $u(r)$ and $v(r)$ are complex functions of $r$ (sums of a scalar and an $I \boldsymbol{\sigma}_{3}$ term). Here the $\psi_{l}^{m}$ are spherical monogenics (see e.g. [18]), which carry the angular dependency of the wavefunction. Specifically, the unnormalised monogenic $\psi_{l}^{m}$ is defined by

$$
\psi_{l}^{m}=\left[(l+m+1) P_{l}^{m}(\cos \theta)-P_{l}^{m+1}(\cos \theta) i \sigma_{\phi}\right] e^{m \phi I \sigma_{3}},
$$

where $l \geq 0,-(l+1) \leq m \leq l$, and $P_{l}^{m}$ are the associated Legendre polynomials.

Substituting (96) into the Dirac equation with the time dependence separated out, and using the properties of the spherical monogenics, we arrive at the coupled radial equations

$$
\left(\begin{array}{cc}
1 & -(2 M / r)^{1 / 2} \\
-(2 M / r)^{1 / 2} & 1
\end{array}\right)\left(\begin{array}{l}
u_{1}^{\prime} \\
u_{2}^{\prime}
\end{array}\right)=\boldsymbol{A}\left(\begin{array}{l}
u_{1} \\
u_{2}
\end{array}\right)
$$

where

$$
\boldsymbol{A}=\left(\begin{array}{cc}
\kappa / r & j(E+m)-(2 M / r)^{1 / 2}(4 r)^{-1} \\
j(E-m)-(2 M / r)^{1 / 2}(4 r)^{-1} & -\kappa / r
\end{array}\right)
$$

$u_{1}$ and $u_{2}$ are the reduced functions defined by

$$
u_{1}=r u \quad u_{2}=j r v,
$$

and the primes denote differentiation with respect to $r$. (We employ the abbreviation $j$ for right-sided multiplication by $I \boldsymbol{\sigma}_{3}$.) 
To analyse (98) we first rewrite it in the equivalent form

$$
(1-2 M / r)\left(\begin{array}{l}
u_{1}^{\prime} \\
u_{2}^{\prime}
\end{array}\right)=\left(\begin{array}{cc}
1 & (2 M / r)^{1 / 2} \\
(2 M / r)^{1 / 2} & 1
\end{array}\right) \boldsymbol{A}\left(\begin{array}{l}
u_{1} \\
u_{2}
\end{array}\right) .
$$

This makes it clear that the equations have regular singular points at the origin and horizon $(r=2 M)$, as well as an irregular singular point at $r=\infty$. To our knowledge, the special function theory required to deal with such equations has not been developed. In the massless case the equations can be manipulated into a second order equation of Heun type [26], but this does not appear to help with the present problem. In the absence of a suitable mathemtical theory, we must either attempt a numerical solution, or look for power series with a limited radius of convergence. We start by considering the latter approach, and look for power-series solutions around the horizon. To this end we introduce the series

$$
u_{1}=\eta^{s} \sum_{k=0}^{\infty} a_{k} \eta^{k}, \quad u_{2}=\eta^{s} \sum_{k=0}^{\infty} b_{k} \eta^{k},
$$

where $\eta=r-2 M$. The index $s$ controls the radial dependence of $\psi$ at the horizon, so represents a physical quantity. To find the values that $s$ can take, we substitute (102) into (101) and set $\eta=0$. This results in the equation

$$
\frac{s}{2 M}\left(\begin{array}{l}
a_{0} \\
b_{0}
\end{array}\right)=\left(\begin{array}{ll}
1 & 1 \\
1 & 1
\end{array}\right)\left(\begin{array}{cc}
\kappa /(2 M) & j(E+m)-(8 M)^{-1} \\
j(E-m)-(8 M)^{-1} & -\kappa /(2 M)
\end{array}\right)\left(\begin{array}{l}
a_{0} \\
b_{0}
\end{array}\right)
$$

Rewriting this in terms of a single matrix and setting its determinant to zero yields the two indicial roots

$$
s=0 \quad \text { and } \quad s=-\frac{1}{2}+4 j M E .
$$

The $s=0$ solution is entirely sensible - the power series is analytic, and nothing peculiar happens at the horizon. If one calculates the conserved current $\psi \gamma_{0} \widetilde{\psi}$ associated with this solution, one finds it is finite and inwardpointing at the horizon, as one would expect. The second root leads to solutions which are ill-defined at the horizon, and have a discontinuity in the current there. This raises some delicate issues as regards the physical meaning of these solutions, some of which are discussed in Section 8 of [24]. If one disregards these problems then, remarkably, it turns out that the discontinuity can be interpreted as the creation of a net outward flux of particles at the horizon, described by a Fermi-Dirac distribution at a temperature given by the Hawking temperature [27]

$$
T=\frac{1}{8 \pi M k_{B}} \text {. }
$$


The value of this temperature comes directly from the imaginary part of the second index $s$ in (104). The surprising feature here is that a FermiDirac distribution is obtained without any of the apparatus of quantum field theory. It turns out that repeating this exercise with a field of integer spin (for example a scalar field, or the electromagnetic field) yields the Hawking temperature again, with a flux described by a Bose-Einstein distribution.

This is obviously an interesting and important area to explore more fully, but here we wish to concentrate instead on the question of the existence of bound state solutions. By analogy with the rejection of unnormalisable solutions in the Hydrogen atom problem, we here reject the solution which is singular at the horizon, and ask whether bound state solutions exist composed wholly of the regular solution, with index $s=0$. Since the wavefunction we are using is described by two complex functions of $r$ (the $u(r)$ and $v(r)$ in equation (96)), there are four real degrees of freedom. Two of these are absorbed when we discard the singular solution, and the remaining two correspond to the amplitude and phase of the regular solution at the horizon. The equations are linear so, without loss of generality, we can fix the parameters to arbitrary values. This means we have no degrees of freedom left in the wavefunction, and can proceed to try to find the (complex) values of energy which lead to normalisable solutions - these will be the eigenfunctions we desire. In the absence of the necessary special function theory, this has been carried out numerically. We hope that one day a more analytic approach may be possible.

The numerical method chosen involved picking trial values for the real and imaginary components of the energy, and then integrating outwards from the horizon as far as possible. What sets the limits to such an integration is that either numerical instability sets in, or the wavefunction density starts diverging exponentially, due to an incorrect (non-eigenvalue) value of $E$ being chosen. The search method involving minimizing the density as a function of the imaginary component of $E$ automatically for each $E_{r}$ over a grid of values of $E_{r}$. Some typical results are shown in Fig. 3. These are for $l=0$, a black hole mass $M=1$, and particle mass $m=0.1$. The $y$-axis is $\log _{10}$ of the wavefunction density, so a dynamic range in selecting eigenvalues of about $10^{4}$ is displayed here.

The search for eigenvalues is aided by searching near regions in $E_{r}$ where the non-relativistic theory would predict a bound state. We would expect the non-relativistic energy found in equation (88) to act as a small correction to the rest mass energy $m c^{2}$, giving

$$
E_{n} \approx m c^{2}-\frac{G M m}{2 a_{0} n^{2}}
$$

where $a_{0}$ is the gravitational Bohr radius (85), and $n$ is the quantum number appropriate in the non-relativistic analysis. For the ground state with $m=$ 


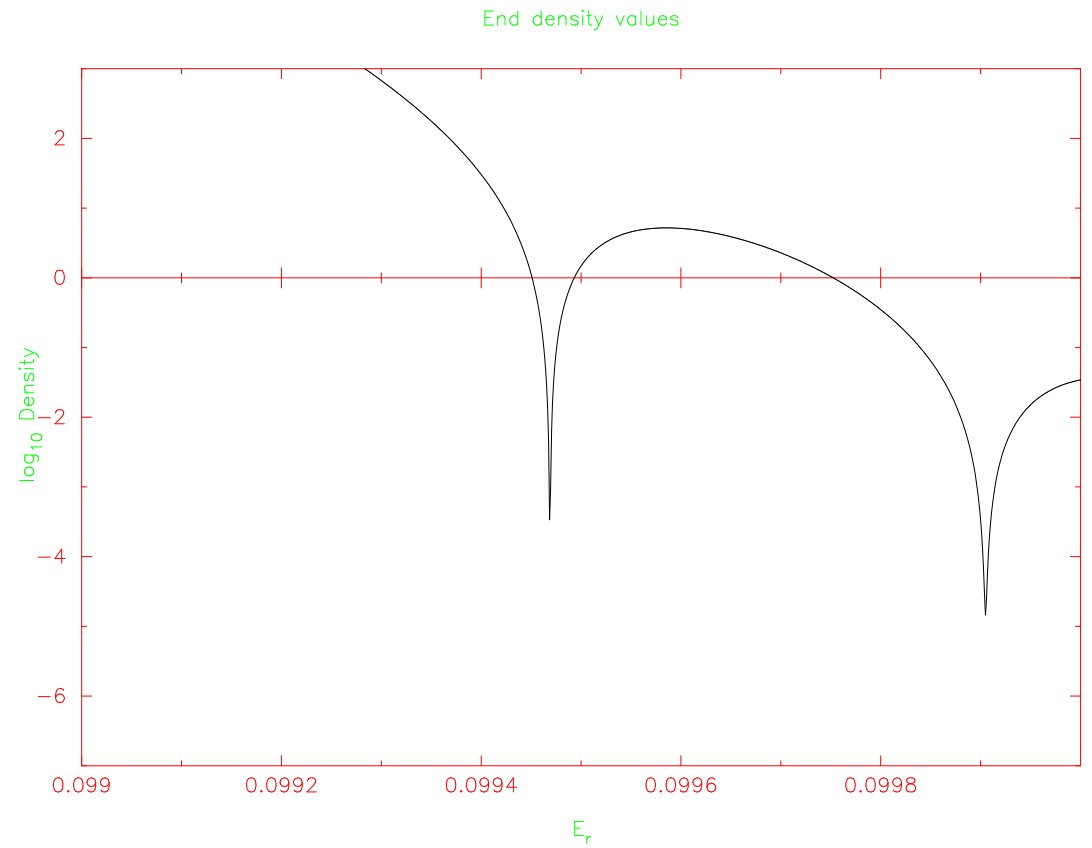

Figure 3. A plot of wavefunction density at a fixed large distance from the black hole, for various values of the real part of the particle energy $E_{r}$, with automatic minimization carried out in $E_{i}$. The particle mass is 0.1 , the angular quantum number $l=0$ and the black hole mass is 1.0. The $y$-axis shows $\log _{10}$ of the wavefunction density at a radius $\left(800 G M / c^{2}\right)$.

$0.1, M=1$, this predicts $E_{r}=0.0995$, indeed very close to where the first dip is seen in Fig. 3. $n=2$ would yield $E_{r}=0.099875$, close to the second dip. The wavefunction density corresponding to this first excited state is shown in Fig. 4.

To establish formally that we have genuine eigenvalues requires integrating out to infinite radial coordinate, and demonstrating that the density integral remains finite. This can be achieved by integrating inwards from infinity and simultaneously outwards from the horizon, and matching at an intermediate point. This process is complicated by the fact that the wavefunction has two types of essential singularity at infinity, which have to be allowed for before series solutions at infinity can be found. One is the $\exp \left(-\sqrt{m^{2}-E^{2}} r\right)$ behaviour which one expects by analogy with the Dirac solution solution for the Hydrogen atom. The second is an essential singularity in the phase, given by a dependence of the form $\exp (j 2 E \sqrt{2 M r})$. A term of this kind could be expected from what we found above for the gravitational Schrödinger equation. 


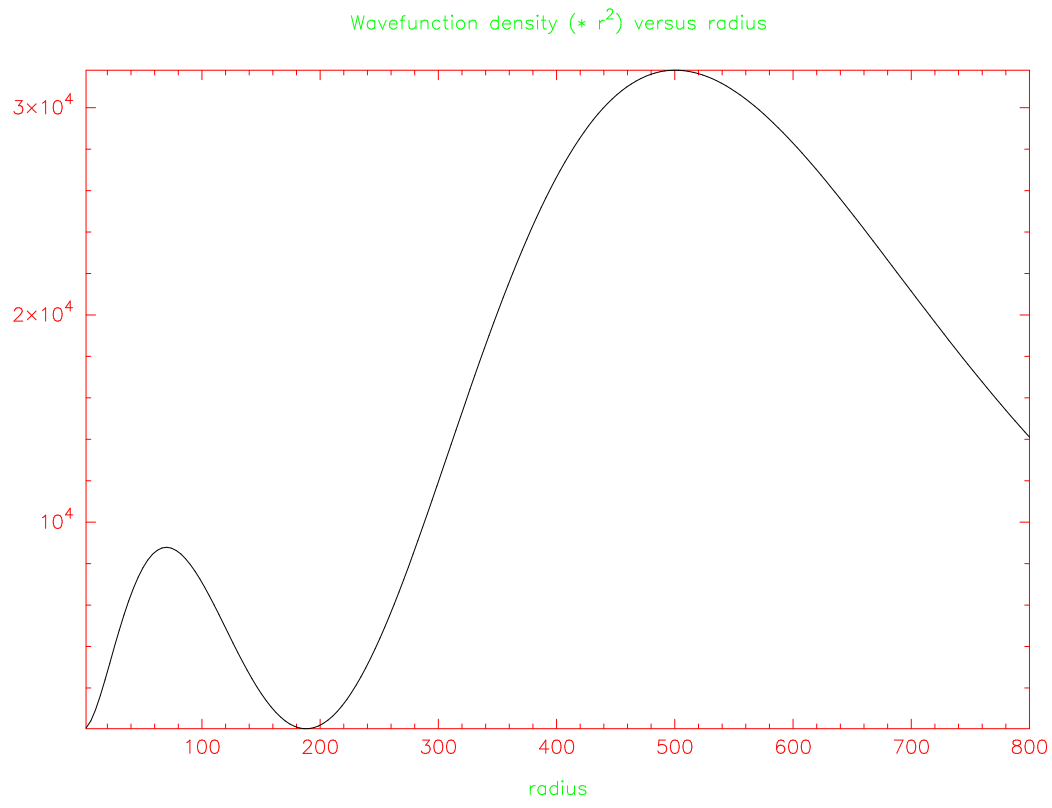

Figure 4. A plot of $r^{2} \times$ wavefunction density for the first excited state of a fermion bound to a black hole. As in Fig. 3 the particle mass is 0.1, the angular quantum number $l=0$ and the black hole mass is 1.0.

TABLE 1. Groundstate energies calculated for two values of $m$ and $M=1$.

\begin{tabular}{|c|c|c|}
\hline$m$ & $E_{r}$ & $E_{i}$ \\
\hline $0.1 \mathrm{e} 0$ & 0.099468827746 & $-2.7870281 \mathrm{e}-6$ \\
$0.2 \mathrm{e} 0$ & 0.194834514694 & -.000751079389 \\
\hline
\end{tabular}

We have confirmed that this process works, which means we can be confident that our eigenvalues are correct. A factor reinforcing confidence is that all the computations have been repeated in a different gauge (the advanced Eddington-Finkelstein gauge - see below) and exactly the same numerical values were found here as well. Furthermore, one can compare the numerically obtained imaginary component of $E$ with that found by integrating the wavefunction to as large a radius as possible and normalising it, and then using equation (93) at the origin. This also checks out precisely. Some ground state eigenvalues are as shown in Table 1 and for the first excited state in Table 2.

What physical regimes might these solutions be interesting in? We can 
TABLE 2. First excited state energies calculated for two values of $m$ and $M=1$.

\begin{tabular}{|c|c|c|}
\hline$m$ & $E_{r}$ & $E_{i}$ \\
\hline 0.1 & 0.099870235768 & $-.356282508 \mathrm{e}-6$ \\
0.2 & 0.19880214433 & -.00009960784655 \\
\hline
\end{tabular}

get some idea of the importance of the decay by defining a dimensionless quantity

$$
\alpha=\frac{\text { gravitational Bohr radius }}{\text { Schwarzschild radius } / 2}
$$

We can re-write this relation in the form

$$
M m=\frac{1}{\sqrt{\alpha}}\left(2.2 \times 10^{-8} \mathrm{~kg}\right)^{2} .
$$

So, for example, if we take $m$ equal to the electron mass, $m=m_{e}=$ $9.1 \times 10^{-31} \mathrm{~kg}$, then $M=(1 / \sqrt{\alpha}) 5.3 \times 10^{14} \mathrm{~kg}$. Primordial black holes of size $10^{12}$ to $10^{14} \mathrm{~kg}$ say, then lie in an interesting range as regards quantum effects.

The size of $\alpha$ is inversely related to the importance of decay effects. A crude estimate, obtained by working with non-relativistic approximations to the ground state wavefunctions, suggests that (in the near nonrelativistic regime) we should find

$$
E_{i} \sim-8 \alpha^{-5 / 2} m c^{2}
$$

which is roughly borne out by the numerical results. For electrons coupled to primordial black holes, this is therefore of order the 'zitterbewegung' time $\hbar /\left(m_{e} c^{2}\right.$ ), (approximately $10^{-21}$ seconds) which is obviously very fast, and it is not clear how such a decay would manifest itself. Typical questions which should now be faced include:

1. What does the detailed energy spectrum look like?

2. What is the significance of the antiparticle solutions, which have opposite sign of the real part of their energy?

3. Do the energy differences between shells correspond to something observable, given the rapid decay rates?

4. If they do, what is the mechanism of radiation, and what quantum jumps are involved?

5. Are there bound states, for which the expectation value of $r$ lies wholly inside the horizon? 
6. What is the extension to the Reissner-Nordstrom and Kerr cases?

7. Can we incorporate multiparticle effects?

The last question is important since a multiparticle approach is necessary to provide a proper link to the Hawking radiation. Equally, in the same way that for the calculation of the Lamb shift it is necessary to evaluate a sum involving bound state energies of the Hydrogen atom, so presumably in the black hole case, vacuum fluctuation effects should be calculated taking proper account of the existence and spectrum of bound states. We are not sure that so far this has been carried out.

\section{Propagators and black hole scattering cross-sections}

We have considered bound states, so an obvious next topic is fermion scattering by black holes. What we wish to look at is again in the spirit of seeing how far we can get in applying conventional quantum mechanics to a gravitational context, using the added facilities of geometric algebra.

In the Coulomb scattering of an electron by a point charge, the first quantum corrections to the Rutherford formula are embodied in the Mott scattering cross-section. A convenient way to arrive at this is via using the Feynman propagator to find the first order $S$-matrix linking initial and final states. In a geometric algebra approach, one finds that the $S$-matrix is replaced by a scaled rotor, which as well as giving the quantum mechanical amplitude, also explicitly embodies the spin transformation properties of the process. In simple cases, this means that the need for spin-sum calculations is obviated, leading to a considerable reduction in the length of the calculations as compared to the conventional approach (see e.g. [18, 28]).

Specifically, let $\psi_{i}(x)$ be an initial 'input' wavefunction and $\psi_{f}(x)$ the final 'output' function and Consider the Coulomb scattering problem to first order. What one finds in the GA approach (see [18] for details) is

$$
\psi_{f}(x)=-S_{f i} \psi_{i}(x) I \sigma_{3} \frac{(2 \pi)^{2}}{E_{f}} \delta\left(E_{f}-E_{i}\right)
$$

where $E_{i}$ and $E_{f}$ are the initial and final energies and $S_{f i}$ is the scaled rotor just referred to. Clearly it rotates from initial to final momentum and spin states, while its magnitude determines the cross-section via

$$
\frac{d \sigma}{d \Omega_{f}}=S_{f i} \widetilde{S}_{f i}
$$

In the case of Coulomb scattering from a nucleus with charge $Z$ one finds explicitly, to first order, that

$$
S_{f i}=\frac{Z \alpha}{\boldsymbol{q}^{2}}(2 E+\boldsymbol{q}) .
$$


Here $\alpha$ is the fine structure constant, $E=E_{i}=E_{f}$ is the electron energy and $\boldsymbol{q}=\boldsymbol{p}_{f}-\boldsymbol{p}_{i}$ is the change in relative momentum. (Recall the relative momentum is defined by $p \gamma_{0}=p \cdot \gamma_{0}+p \wedge \gamma_{0}=E+\boldsymbol{p}$.). This leads to the Mott cross-section

$$
\frac{d \sigma}{d \Omega_{f}}=\frac{Z^{2} \alpha^{2}}{\boldsymbol{q}^{4}}\left(4 E^{2}-\boldsymbol{q}^{2}\right)=\frac{Z^{2} \alpha^{2}}{4 \boldsymbol{p}^{2} \beta^{2} \sin ^{4}(\theta / 2)}\left(1-\beta^{2} \sin ^{2}(\theta / 2)\right)
$$

where

$$
\boldsymbol{q}^{2}=\left(\boldsymbol{p}_{f}-\boldsymbol{p}_{i}\right)^{2}=2 \boldsymbol{p}^{2}(1-\cos \theta) \quad \text { and } \quad \beta=|\boldsymbol{p}| / E .
$$

Now the interaction Hamiltonian for the Coulomb potential is the simple scalar operator

$$
H_{C} \psi=\frac{Z e^{2}}{4 \pi \epsilon_{0} r} \psi
$$

while for an electron interacting with a black hole using the Newtonian gauge we have seen the interaction Hamiltonian has the form

$$
H_{I} \psi=\left(\frac{2 G M}{r}\right)^{1 / 2} \hbar\left(\partial_{r} \psi+3 /(4 r) \psi\right) I \sigma_{3}
$$

The essential part of this is another scalar operator, but this time with a derivative in $r$ present in it. The simplicity of this form suggests we ask whether we can employ the same Feynman propagator techniques we used in the Coulomb calculation to obtain an analogue of the Mott scattering formula in the gravitational case. Along the way we would find the gravitational analogue of the 'rotor' $S_{f i}$, which would again have the benefit of obviating the need for spin sums.

The key part of the derivation will be finding the momentum space representation of $H_{I}$. We may write schematically

$$
\mathcal{H}_{I}\left(\boldsymbol{p}_{2}, \boldsymbol{p}_{1}\right)=\int d^{3} x e^{-j \boldsymbol{p}_{2} \cdot \boldsymbol{x}} \mathcal{H}_{I} e^{j \boldsymbol{p}_{1} \cdot \boldsymbol{x}}
$$

which yields

$$
\mathcal{H}_{I}\left(\boldsymbol{p}_{2}, \boldsymbol{p}_{1}\right)=\frac{j 3 \pi^{3 / 2}(G M)^{1 / 2}}{\left|\boldsymbol{p}_{2}-\boldsymbol{p}_{1}\right|^{7 / 2}}\left(\boldsymbol{p}_{2}^{2}-\boldsymbol{p}_{1}^{2}\right) .
$$

On multiplying this by $\gamma_{0}$ we obtain the desrired vertex factor. This has the unusual property of vanishing on-shell, when $\left|\boldsymbol{p}_{1}\right|=\left|\boldsymbol{p}_{2}\right|$. (Energy is conserved throughout since the interaction Hamiltonian is independent of time). In retrospect, this is as we might expect, since otherwise we would have the matrix element going as $\sqrt{M}$ in leading order, rather than as $M$, which is what what we would expect on the basis of the analogy with $Z$ in the Coulomb case. 
While this makes sense, it means we have to go to second order in our calculations, which is a perhaps unfortunate feature of the Newtonian gauge. It is thus of interest to find an alternative gauge in which the desired scattering result can emerge at first order. If this answer agrees with that from the second order Newtonian gauge answer, this would be a powerful indication that both are right and that the result we are calculating is physically meaningful (gauge invariant).

The suitable gauge in which to attempt this is the advanced Eddington Finkelstein (AEF) gauge. This has as its h-function

$$
\overline{\mathrm{h}}(a)=a+\frac{M}{r} a \cdot n n \quad \text { where } \quad n=\gamma_{0}-\hat{r}
$$

and $\hat{r}$ is a unit spacelike vector in the radial direction, so that $n$ is null. This h-function has a simple form, and the gravitational effects of the black hole enter linearly in $M$, as we want, rather than proportional to $\sqrt{M}$ as in the Newtonian gauge case. However, unlike the latter case, the interaction Hamiltonian is no longer a simple scalar operator on $\psi$, but has a multivector structure, not analogous to the Coulomb case. Specifically, the Dirac equation in this gauge is

$$
\nabla \psi I \boldsymbol{\sigma}_{3}+\frac{G M}{r}\left(\gamma_{0}-\hat{r}\right)\left(\frac{\partial}{\partial t}-\frac{\partial}{\partial r}-\frac{1}{2 r}\right) \psi I \boldsymbol{\sigma}_{3}=m \psi \gamma_{0}
$$

We can still apply the Feynman techniques even in this case, however, by using the following route. For the general Dirac equation in a gravitational field,

$$
D \psi I \sigma_{3}-m \psi \gamma_{0}=0
$$

let us rewrite it instead as

$$
\nabla \psi i \sigma_{3}-m \psi \gamma_{0}=\nabla \psi i \sigma_{3}-D \psi i \sigma_{3}
$$

This appears to be a trivial rewriting, but means that we can view the right hand side as an interaction term which can be solved for using the freeparticle Feynman propagator appropriate to the left hand side. This is quite a powerful technique, and will apply wherever there are asymptotically free in and out states. Some details of the evaluation of the matrix element in this case are discussed in [6], but here we simply state the result:

$$
S_{f i}=\frac{G M}{\boldsymbol{q}^{2}}\left(E(2 E+\boldsymbol{q})+\boldsymbol{p}^{2}+\boldsymbol{p}_{f} \boldsymbol{p}_{i}\right)
$$

This is the scaled rotor which transforms between initial and final states for a fermion scattering from a black hole, to first order in $M$, calculated 
using the AEF gauge. This is to be compared and contrasted with the equivalent Coulomb matrix element (112). It is similar in the first term, though with an extra factor of $E$, and then contains two extra terms. We note immediately that the presence of the extra $E$ in the first term is a manifestation of the equivalence principle! Without it, the cross-section would depend explicitly on the particle mass $m$. With it, the cross-section is a function of the particle velocity only.

Before continuing to discuss the cross-section coming from this matrix element, we have to satisfy ourselves that it is physically meaningful. Here we have the advantage that we can verify that we obtain the same result using the Newtonian gauge to second order. The details are given in [6] but it turns out that indeed the result is identical to (123), which helps verify gauge invariance. A further argument in favour of gauge invariance is that the similar computation in the Coulomb case is definitely gauge invariant - if we replace the electromagnetic potential $A$ with $A-\nabla \alpha(r)$, so that $A \mapsto A+\hat{r} d \alpha / d r$, one finds that (on-shell) the matrix element $S_{f i}$, and therefore the cross-section, is unaffected. A final piece of evidence is that we can be certain that in the AEF gauge we will not obtain an effect at order $M^{3 / 2}$, since the perturbation series will involve whole powers of $M$. This predicts that the third order effect in the Newtonian gauge should vanish. This can be shown to be indeed the case by explicit computation in the Newtonian gauge. We therefore take it that our result is physically meaningful, although an explicit proof of gauge invariance (ideally to all orders in $M$ ) would of course be desirable.

The cross-section following from (112) is

$$
\frac{d \sigma}{d \Omega_{f}}=\frac{(G M)^{2}}{4 \beta^{4} \sin ^{4}(\theta / 2)}\left(1+2 \beta^{2}-3 \beta^{2} \sin ^{2} \frac{\theta}{2}+\beta^{4}-\beta^{4} \sin ^{2} \frac{\theta}{2}\right)
$$

and is plotted for a particular case in Figure 5. It is possible to check our result against the classical limit for black hole scattering by comparing with the result of Collins, Delbourgo and Williams in [1]. They were able to obtain an explicit series solution for the classical cross-section in the limit of small $\theta$. The first term in their series is of order $M^{2}$ and is (translating to our notation)

$$
\frac{d \sigma}{d \Omega}=\left(\frac{2 G M\left(2 \gamma^{2}-1\right)}{\theta^{2}\left(\gamma^{2}-1\right)}\right)^{2}
$$

where $\gamma$ is the Lorentz factor $\left(1-\beta^{2}\right)^{-1 / 2}$. This agrees precisely with the first term in the small angle expansion of our result, equation (124). Their second term

$$
\frac{3 \pi(G M)^{2}\left(5 \gamma^{2}-1\right)}{4 \theta^{3}\left(\gamma^{2}-1\right)}
$$




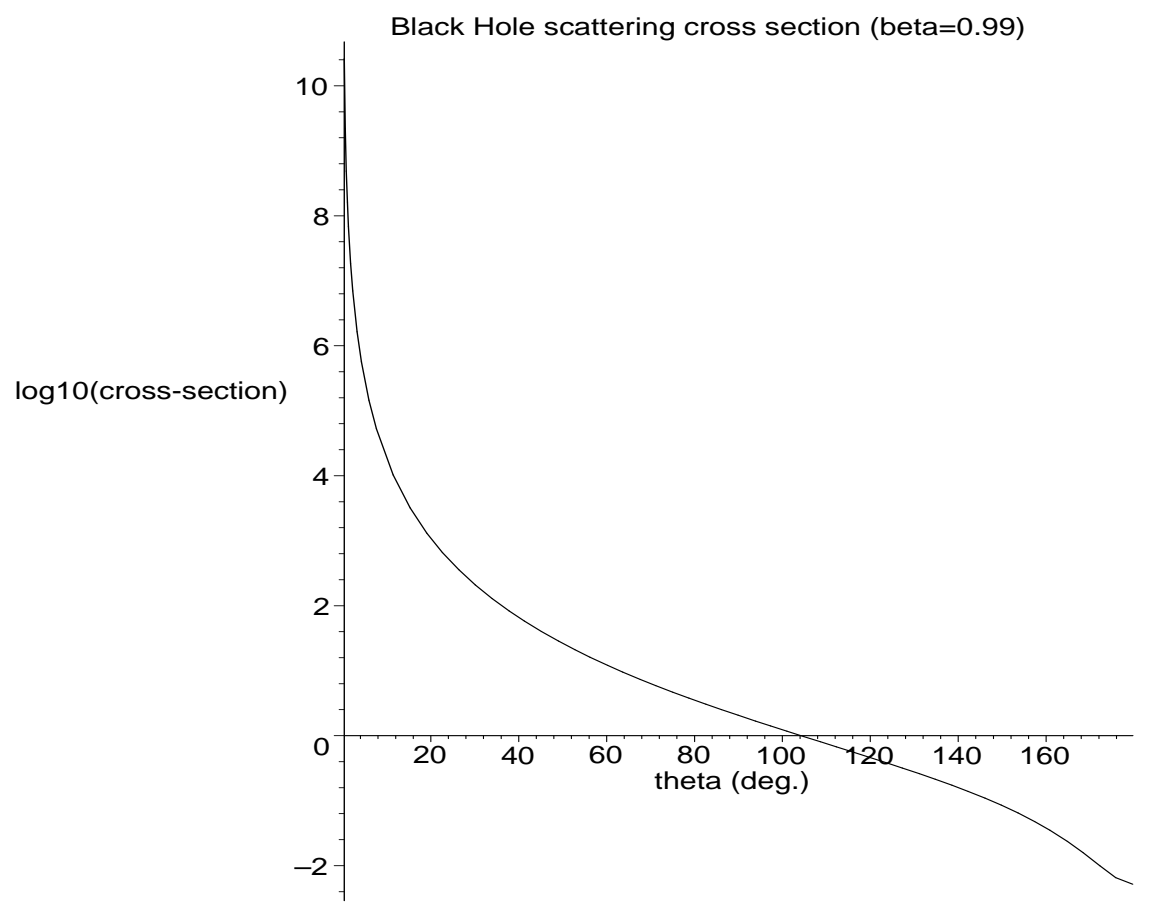

Figure 5. Plot of the black hole scattering cross-section formula (124) for a high speed particle $(\beta=0.99)$, and with $M=1$.

is also of order $M^{2}$, and therefore might be expected to agree with us also. However, our second term is

$$
-\frac{(G M)^{2}\left(4 \gamma^{4}-7 \gamma^{2}+1\right)}{3 \theta^{2}\left(\gamma^{2}-1\right)^{2}}
$$

which as well as being quite different numerically, is also different as regards its order in $\theta$, going as $\theta^{-2}$ rather than $\theta^{-3}$. We cannot explain this discrepancy, and indeed it seems strange that an odd power of $\theta$ could be obtained at all, even in the classical result.

The massless limit $m \mapsto 0$ is also well-defined and leads to the simple formula

$$
\frac{d \sigma}{d \Omega}=\frac{(G M)^{2} \cos ^{2}(\theta / 2)}{\sin ^{4}(\theta / 2)} .
$$

Again, the low-angle limit recovers the classical formula for the bending of light. This result also predicts zero amplitude in the backward direction, $\theta=$ $\pi$. Null geodesics produce a significant flux in the backward direction, and the fact that zero is predicted here is a diffraction effect for neutrinos which 
goes beyond the predictions of geometric optics. A similar prediction of zero back-scattering for neutrinos was made in [3]. A more detailed analysis of the cross section in the backward direction also reveals a large 'glory' scattering $[3,5]$. In the geometric optics limit this is attributable to multiple orbits, and in the quantum description the glory scattering is described by higher-order terms in GM. To describe these effects in the present scheme requires extending to higher order in perturbation theory. This is currently under investigation.

Extending to higher orders also raises the question of the convergence of the iterative scheme proposed here. This is not a straightforward issue to address as there is no dimensionless coupling constant in the problem. Also, it is not clear whether higher-order quantum terms should still be expected to obey the equivalence principle. One can easily formulate desirable criteria for convergence, such as $G M E<1$ or $G M E v<1$, but these are too restrictive, given that the low angle formula we arrive at is expected to be valid for all masses and velocities. It would appear that the only way to investigate convergence is to compute the next order terms in the perturbation series directly.

This work should also have clarified the importance of working consistently to the correct order in $M$. This is particularly clear in the Schwarzschild gauge, where the interaction term contains factors which go as 1 $(1-2 G M / r)^{1 / 2}$. An iterative scheme based on this gauge choice should expand out the vertex factor as a series in $M$, and then keep all of the terms up to the desired order. Such a scheme is workable, but has the disadvantage of introducing new vertex terms at each order in the series solution. It is straightforward to confirm that such a scheme reproduces our result for the fermion cross section, to lowest order.

The next steps include the following:

1. Extension of the above results to second order in the AEF gauge (and perhaps 4th order in Newtonian gauge, to verify gauge invariance at order $M^{2}$ ). Potential new features which may emerge include the 'glory' back scattering effect, mentioned above ([5]).

2. Extension to the Riessner-Nordstrom and Kerr cases. The interaction with the angular momentum in the Kerr case will be particularly interesting, but the computations currently look difficult.

3. Another vertex which can be tried is interaction with a photon, in the presence of the background gravitational field, in order to calculate the gravitational equivalent of bremsstrahlung. This could shed some light on the long-standing problem of the radiation caused by a freely falling electron. 


\section{Riemann-squared theory and torsion effects in the early uni- verse}

We would like finally to discuss some progress in applying GA techniques to higher-order Lagrangian field theories. Our group has already looked at the effects of torsion in gauge theory gravity, and found a new solution for the Dirac field coupled self-consistently to gravity in which torsion was important $[29,30]$. We have also considered a GA approach to quadratic gravitational Lagrangians and to topological invariants, in [31]. Here, we wish to highlight some recent work which may be important in application to the early universe.

The higher-order Lagrangian theory which is perhaps best motivated in analogy with other gauge theories is one where the Lagrangian is given by the same expression as would be used in any Yang-Mills theory - the field strength tensor squared. Written in the GA approach this is a term in

$$
\mathcal{L}_{2}=\mathcal{R}\left(e^{\mu} \wedge e^{\nu}\right) \cdot \mathcal{R}\left(e_{\nu} \wedge e_{\mu}\right) .
$$

This has been explored several times and is known to lead to spherically symmetric metrics of Schwarzschild-like form but with

$$
\left(1-\frac{2 M}{r}+\kappa r^{2}\right) \quad \text { in place of }\left(1-\frac{2 M}{r}\right)
$$

If one demands asymptotic flatness, then $\kappa=0$ and one is back with the standard Schwarzschild metric, which means that the theory passes all the standard solar system tests.

A key feature of this type of theory, which makes it particularly attractive, is that it satisfies scale covariance as well as position and rotation gauge covariance. It thus encompasses all the symmetries one might expect to be present. A further feature is that it includes torsion. This is the case even if there is no source of quantum spin, which is a significant difference from theories using the using a Ricci scalar Lagrangian for the gravitational field. For these the torsion is generated entirely by matter. In cosmology, for example, we can consider coupling self-consistently with a Dirac field, which is the form of torsion considered in [29] and [30].

Working with the Lagrangian $\mathcal{L}_{2}$ we can derive the field equations appropriate for cosmology. In what follows we have assumed spatial flatness. The equations yields a remarkable result. Writing

$$
A=2 H^{2}+\dot{H}-\frac{1}{4} Q^{2} \quad \text { and } \quad B=\frac{3}{2} Q H+\frac{1}{2} \dot{Q}
$$

where $H$ is the Hubble parameter and $Q$ is a measure of the 'free-space' torsion (both real), we find that it is possible to write the evolution equations 


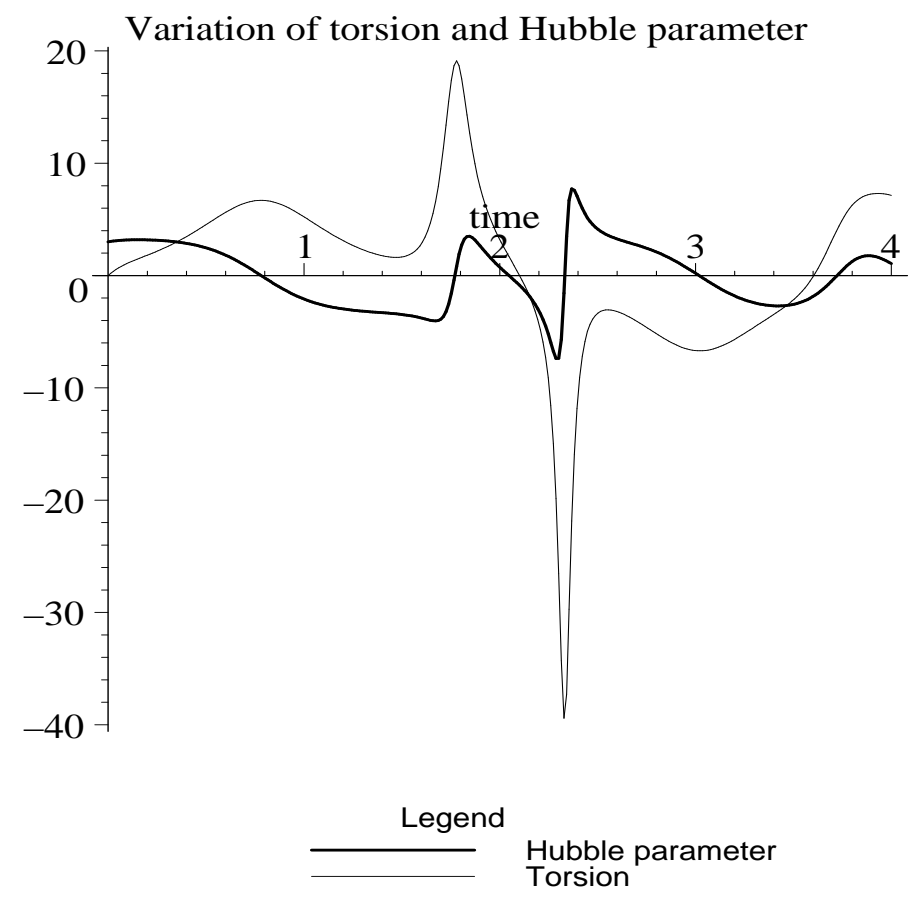

Figure 6. Illustrative plot of variation of Hubble parameter $(H)$ and and free-space torsion $(Q)$ with $Q$ starting at small values. In this case, the matter density is zero at all times, so it is only the torsion field providing the dynamics.

together jointly in a complex form:

$$
\frac{d}{d t}(A+i B)=i Q(A+i B)
$$

Thus the modulus of the quantity $(A+i B)$ is constant, but its phase gets driven round in a potentially chaotic fashion by the torsion, $Q$. These are the full equations of the cosmology. They reduce to equations people have studied before in Riemann squared cosmology for $Q=0$, but it is not clear whether the above behaviour is known for the torsion case.

If one computes numerically some typical results, one finds that the regular behaviour of $H$ is disturbed by the torsion $Q$ which, provided it is not precisely zero initially, builds up and then forces a rapid change in $H$. $Q$ then declines in value again, before another episode of building up. A typical example is shown in Figure 6. This is actually computed for zero matter density, and shows that the addition of torsion by itself is quite sufficient to give some interesting dynamics. A problem for the matter sector of any scale invariant theory, is that the matter stress-energy tensor has to 
have zero trace. This precludes using normal matter, and means that such cosmologies are probably unrealistic. One of the main current cosmological problems, however, is how to generate a period of inflation in the early universe, and then a hugely smaller effective cosmological constant today. It is certainly interesting that torsion can give somewhat chaotic behaviour of the type illustrated, with very large swings of the Hubble parameter, unrelated to the underlying matter field.

\section{Acknowledgements}

ANL would like to thank Venzo de Sabbata for the opportunity of attending the meeting in Erice and contributing to this volume. CJLD is supported by the EPSRC.

\section{References}

1. P.A. Collins, R. Delbourgo, and R. M. Williams. On the elastic Schwarzschild scattering cross section. J. Phys. A, 6:161-169, 1973.

2. N. Sanchez. Elastic scattering of waves by a black hole. Phys. Rev. D, 18(6):1798$1804,1978$.

3. J.A.H. Futterman, F.A. Handler, and R.A. Matzner. Scattering from Black Holes. Cambridge University Press, 1988.

4. S. Chandrasekhar. The Mathematical Theory of Black Holes. Oxford University Press, 1983.

5. N. Andersson and B.P. Jensen. Scattering by black holes. In R. Pike and P. Sabatier, editors, Scattering. Academic Press, 2000. See also gr-qc/0011025.

6. C.J.L. Doran and A.N. Lasenby. Perturbation theory calculation of the black hole elastic scattering cross section. To appear in: Physical Review D (gr-qc/0106039), 2001.

7. D. Hestenes. Vectors, spinors, and complex numbers in classical and quantum physics. Am. J. Phys., 39:1013, 1971.

8. D. Hestenes. Proper particle mechanics. J. Math. Phys., 15(10):1768, 1974.

9. D. Hestenes. Observables, operators, and complex numbers in the Dirac theory. $J$. Math. Phys., 16(3):556, 1975.

10. T.G. Vold. An introduction to geometric algebra with an application to rigid body mechanics. Am. J. Phys., 61(6):491, 1993.

11. T.G. Vold. An introduction to geometric calculus and its application to electrodynamics. Am. J. Phys., 61(6):505, 1993.

12. D. Hestenes and G. Sobczyk. Clifford Algebra to Geometric Calculus. Reidel, Dordrecht, 1984.

13. D. Hestenes. New Foundations for Classical Mechanics (Second Edition). Kluwer Academic Publishers, Dordrecht, 1999.

14. C.J.L Doran and A.N. Lasenby. Geometric Algebra for Physicists. Cambridge University Press, 2001. In press.

15. C.J.L. Doran, A.N. Lasenby, S.F. Gull, and J. Lasenby. Lectures in geometric algebra. In W.E. Baylis, editor, Clifford (Geometric) Algebras, pages 65-236. Birkhauser, Boston, 1996.

16. C.J.L. Doran and A.N. Lasenby. Physical applications of geometric algebra. Lecture notes available from http://www.mrao.cam.ac.uk/ clifford.

17. C.J.L. Doran, A.N. Lasenby, and S.F. Gull. States and operators in the spacetime algebra. Found. Phys., 23(9):1239, 1993. 
18. C.J.L Doran, A.N. Lasenby, S.F. Gull, S.S. Somaroo, and A.D. Challinor. Spacetime algebra and electron physics. Adv. Imag. Es Elect. Phys., 95:271, 1996.

19. S.S. Somaroo, A.N. Lasenby, and C.J.L. Doran. Geometric algebra and the causal approach to multiparticle quantum mechanics. J. Math. Phys., 40(7):3327-3340, 1999.

20. T.F. Havel and C.J.L. Doran. Geometric algebra in quantum information processing. quant-ph/0004031, AMS Contemporary Math series, to appear.

21. S. Somaroo, D.G. Cory, and T.F. Havel. Expressing the operations of quantum computing in multiparticle geometric algebra. Phys. Lett. A, 240:1-7, 1998.

22. T.W.B. Kibble. Lorentz invariance and the gravitational field. J. Math. Phys., 2(3):212, 1961.

23. F.W. Hehl, P. von der Heyde, G.D. Kerlick, and J.M. Nester. General relativity with spin and torsion: Foundations and prospects. Rev. Mod. Phys., 48:393, 1976.

24. A.N. Lasenby, C.J.L. Doran, and S.F. Gull. Gravity, gauge theories and geometric algebra. Phil. Trans. R. Soc. Lond. A, 356:487-582, 1998.

25. K. Martel and E. Poisson. Regular coordinate systems for Schwarzschild and other spherical spacetimes. Am. J. Phys., 69(4):476, 2001.

26. A. Ronveaux (ed.). Heun's Differential Equations. Oxford University Press, 1995.

27. S. W. Hawking. Black hole explosion? Nature, 248:30, 1974.

28. A.M. Lewis, C.J.L. Doran, and A.N. Lasenby. Electron scattering without spin sums. Int. J. Theor. Phys., 40(1), 2001.

29. C.J.L Doran, A.N. Lasenby, A.D. Challinor, and S.F Gull. Effects of spin-torsion in gauge theory gravity. J. Math. Phys., 39(6):3303, 1998.

30. A.D. Challinor, A.N. Lasenby, C.J.L Doran, and S.F Gull. Massive, non-ghost solutions for the Dirac field coupled self-consistently to gravity. General Rel. Grav., 29:1527, 1997.

31. A.M. Lewis, C.J.L. Doran, and A.N. Lasenby. Quadratic Lagrangians and topology in gauge theory gravity. Gen. Rel. Grav., 32(1):161, 2000. 